\title{
Opacity of Shock-Heated Boron Plasmas
}

\author{
W. R. Johnson \\ University of Notre Dame, Notre Dame, IN 46556 \\ J. Nilsen \\ Lawrence Livermore National Laboratory, Livermore, CA 94551
}

\begin{abstract}
Standard measures of opacity, the imaginary part of the atomic scattering factor $f_{2}$ and the x-ray mass attenuation coefficient $\mu / \rho$, are evaluated in shock-heated boron, boron carbide and boron nitride plasmas. The Hugoniot equation, relating the temperature $T$ behind a shock wave to the compression ratio $\rho / \rho_{0}$ across the shock front, is used in connection with the plasma equation of state to determine the pressure $p$, effective plasma charge $Z^{*}$ and the $\mathrm{K}$-shell occupation in terms of $\rho / \rho_{0}$. Solutions of the Hugoniot equation (determined within the framework of the generalized Thomas-Fermi theory) reveal that the K-shell occupation in low- $\mathrm{Z}$ ions decreases rapidly from 2 to 0.1 as the temperature increases from $20 \mathrm{eV}$ to $500 \mathrm{eV}$; a temperature range in which the shock compression ratio is near 4 . The average-atom model (a quantum mechanical version of the generalized Thomas-Fermi theory) is used to determine K-shell and continuum wave functions and the photoionization cross section for $\mathrm{x}$-rays in the energy range $\omega=1 \mathrm{eV}$ to $10 \mathrm{keV}$, where the opacity is dominated by the atomic photoionization process. For an uncompressed boron plasma at $T=10 \mathrm{eV}$, where the K-shell is filled, the average-atom cross section, the atomic scattering factor and the mass attenuation coefficient are all shown to agree closely with previous (cold matter) tabulations [1-3]. For shock-compressed plasmas, the dependence of $\mu / \rho$ on temperature can be approximated by scaling previously tabulated cold-matter values by the relative $\mathrm{K}$-shell occupation; however, there is a relatively small residual dependence arising from the photoionization cross section. Attenuation coefficients $\mu$ for a $9 \mathrm{keV}$ x-ray are given as functions of $T$ along the Hugoniot for $\mathrm{B}, \mathrm{C}, \mathrm{B}_{4} \mathrm{C}$ and $\mathrm{BN}$ plasmas.
\end{abstract}

Keywords: 52.50.Lp: Plasma production and heating by shock waves and compression, 56.65.Rr: Particle in cell method, 52.70.-m: Plasma diagnostic techniques, 52.25.Os: Emission, absorption, and scattering of electromagnetic radiation.

Email addresses: johnson@nd.edu (W. R. Johnson), nilsen1@llnl.gov (J. Nilsen) 


\section{Introduction}

The aim of this paper is to examine the opacity of the hot dense plasma behind an intense shock wave impinging on matter at standard temperature and pressure. For simplicity, the present study is restricted to boron, carbon and two boron compounds, boron carbide and boron nitride; however, the methods developed herein are applicable to other light-ion plasmas such as polystyrene.

A brief review of shock waves and the Hugoniot equation, which governs the thermodynamic properties of the plasma behind a shock front, is given first. This discussion is followed by a review of the generalized Thomas-Fermi (TF) theory of Feynman, Metropolis and Teller [4, which is used to determine the energy per ion and pressure on the two sides of shock front in terms of the respective temperatures and densities. With such information, the Hugoniot equation can be solved to give the temperature $T$, pressure $p$, effective ionic charge $Z^{*}$ and the occupation of the ionic K-shell as functions of the compression ratio $\rho / \rho_{0}$.

The K-shell occupation, which is a critical factor in determining the plasma opacity, is determined by solving the Schrödinger equation in the TF potential. Such a procedure is based on the assumption that the semi-classical TF potential is a good approximation to a proper quantum-mechanical potential. To test this assumption, the $\mathrm{K}$-shell occupation is re-evaluated using a quantum-mechanical average-atom cell model to describe the plasma. Differences of $10 \%$ or less are found between the semi-classical and quantum-mechanical determinations of the K-shell occupation numbers.

Following this comparison, we introduce the (complex) atomic scattering factor $f(\theta)$ together with the relation between the index of refraction $n$ in a medium and the forward scattering factor $f(0)$. The forward-scattering factor $f(0)$ is tabulated as a function of photon energy by Henke, et al. [2] for (cold) elements $1 \leq Z \leq 92$. The imaginary part $f_{2}\left(f(0)=f_{1}-i f_{2}\right)$, which is a function of the photon energy $\omega$, is responsible for the exponential decay of the intensity of an electromagnetic wave. In the x-ray region beyond the $\mathrm{K}$-shell threshold $f_{2}$ is proportional to the atomic bound-free photoionization cross section $\sigma_{\mathrm{bf}}(\omega)$.

The average-atom model is used to evaluate the photoionization cross section $\sigma_{\text {bf }}(\omega)$ for energies above the K-shell threshold $(\approx 200 \mathrm{eV}$ for boron) to $10 \mathrm{keV}$. It should be noted that average-atom models in various guises have been used previously to study photoionization and opacity in warm- and hot-dense plasmas [5] 15. The photoionization cross section calculated using the present model for a boron plasma at temperature $T=10 \mathrm{eV}$ and density $\rho=2.463 \mathrm{~g} / \mathrm{cc}$, where the $\mathrm{K}$-shell is filled, is in close agreement with the boron K-shell cross section given in the tabulation of Yeh and Lindau [1. With the aid of the relation between $f_{2}$ and $\sigma_{\mathrm{bf}}$, one determines $f_{2}$ for shock-compressed plasmas. Close agreement is found between the resulting average-atom calculations of $f_{2}$ and values in the Henke tabulation for a boron plasma at temperature $T=10 \mathrm{eV}$ and density $\rho=2.463 \mathrm{~g} / \mathrm{cc}$.

Finally, the x-ray mass attenuation coefficient $\mu$, tabulated for cold matter 
by Hubbell and Seltzer [3], is introduced and expressed in terms of the $\sigma_{\mathrm{bf}}$. The coefficient $\mu$, which is the reciprocal of the $1 / e$ x-ray intensity falloff distance, is proportional to the plasma density; the ratio $\mu / \rho$ for $9 \mathrm{keV}$-rays is found to be strongly dependent on the K-shell occupation in the $20-500 \mathrm{eV}$ temperature range and weakly dependent on the remaining factors governing the photoionization cross section. Plots are presented illustrating $\mu(\omega)$ for a $9 \mathrm{keV}$ He-like Zn x-ray as a function of $T$ behind a shock moving into cold $B, C, B_{4} C$ and $B N$ plasmas.

\section{Shock Waves and the Hugoniot Equation}

A shock wave, illustrated in Fig. 1, moving with velocity $u_{s}$ into a (cold) medium at rest having pressure $p_{0}$, density $\rho_{0}$, energy per ion $e_{0}$ and temperature $T_{0}$ leaves behind a hot compressed medium with pressure $p$, density $\rho$, energy $e$ and temperature $T$ moving with velocity $u$. These quantities are subject to the conservation laws (formulated for a plane shock wave in a reference frame moving with the shock):

$$
\begin{aligned}
\rho\left(u_{s}-u\right) & =\rho_{0} u_{s} & \text { Continuity } \\
p+\rho\left(u_{s}-u\right)^{2} & =p_{0}+\rho u_{s}^{2} & \text { Conservation of Momentum } \\
e+\frac{1}{2}\left(u_{s}-u\right)^{2} & =e_{0}+\frac{1}{2} u_{s}^{2} & \text { Conservation of Energy }
\end{aligned}
$$

Determining $u_{s}$ and $u$ from the first two equations leads to the following reformulation of the law of conservation of energy:

$$
H(T, \rho)=\left(e-e_{0}\right)-\frac{1}{2}\left(p+p_{0}\right)\left(\frac{1}{\rho_{0}}-\frac{1}{\rho}\right)=0 .
$$

Eq.(1), referred to as the Hugoniot equation, can be solved to obtain both the temperature $T$ and the pressure $p$ in the shocked region in terms of the density ratio $\rho / \rho_{0}$, assuming that the thermodynamic properties of the unshocked medium are known.

The density $\rho$ and pressure $p$ in the shocked medium can be determined experimentally as illustrated in Ref. 13. from knowledge of the density $\rho_{0}$ and pressure $p_{0}$ in the un-compressed medium and measurements of $u$ and $u_{s}$ using the following relations that can be inferred from the conservation laws:

$$
\begin{aligned}
\rho / \rho_{0} & =u_{s} /\left(u_{s}-u\right) \\
p & =p_{0}+\left(1-\rho_{0} / \rho\right) \rho_{0} u_{s}^{2} .
\end{aligned}
$$

In the present work, we determine the contribution of plasma electrons to $H(T, \rho)$ using the generalized Thomas-Fermi theory of Feynman, Metropolis and Teller 4 and determine contributions of ions to $H(T, \rho)$ using the ideal gas law. 


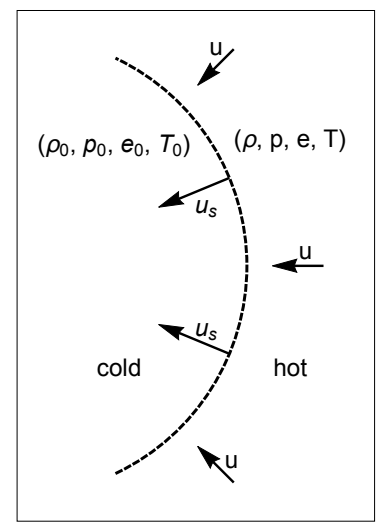

Figure 1: A shock wave, illustrated by the dashed arc, moves with velocity $u_{s}$ into a cold medium, leaving behind a hot compressed medium moving with velocity $u$.

\section{Generalized Thomas-Fermi Theory}

The generalized Thomas-Fermi theory is a well-known method for evaluating the electron pressure $p$, chemical potential $\mu$, free electron number density $n_{e}$ and energy $e$ in terms of temperature $T$ and density $\rho$. In this theory, a neutral plasma consisting of ions of nuclear charge $Z$ and electrons is divided into neutral Wigner-Seitz (WS) cells of volume $V_{\mathrm{WS}}=A /\left(N_{A} \rho\right)$, where $A$ is the atomic weight of the ion and $N_{A}$ is Avogadro's number. Inside the WS cell, electrons are assumed to move in a self-consistent potential:

$$
V(r)=-\frac{Z}{r}+\int_{r^{\prime} \leq R} \frac{n_{e}\left(\boldsymbol{r}^{\prime}\right) d^{3} r^{\prime}}{\left|\boldsymbol{r}-\boldsymbol{r}^{\prime}\right|},
$$

where $R$ is the radius of the WS sphere. The electron density $n_{e}(r)$ in Eq. (4) is given by the semi-classical expression:

$$
n_{e}(r)=\frac{1}{\pi^{2}} \int_{0}^{\infty} \frac{p^{2} d p}{1+\exp \left[\left(p^{2} / 2 m+V(r)-\mu\right) / k T\right]},
$$

where $p$ represents electron momentum. The chemical potential $\mu$ is determined by the requirement that the cell be neutral:

$$
\int_{r \leq R} n_{e}(r) d^{3} r=Z
$$

Equations (4 6) are solved self-consistently to give the electron density inside the WS cell, the potential $V(r)$ and the chemical potential $\mu$. The potential $V(r)$ satisfies

$$
V(R)=0 \quad \text { and } \quad \frac{d V(R)}{d r}=0 .
$$



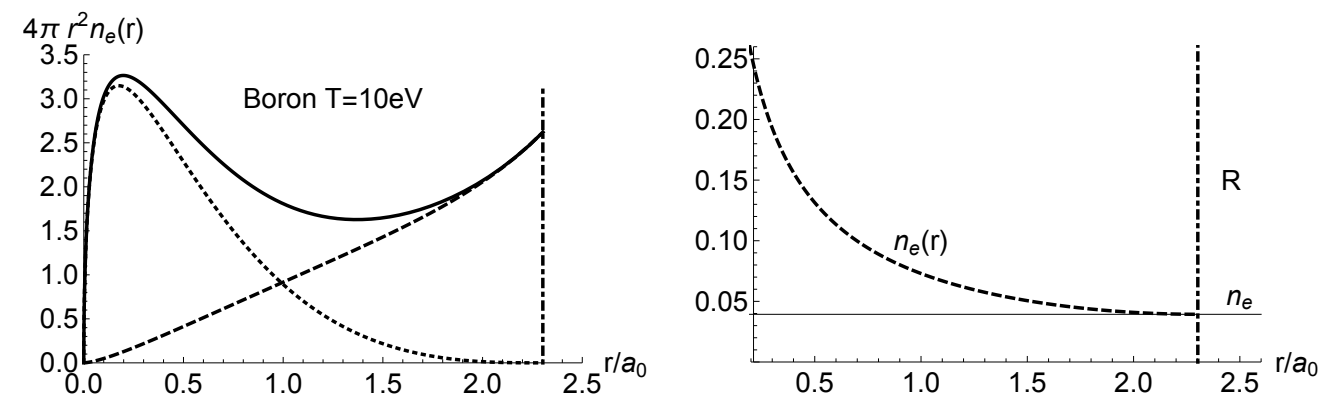

Figure 2: Left panel: The solid curve shows the radial electron number density $4 \pi r^{2} n_{e}(r)$ obtained by solving the TF Eqs. 4.6) for a boron plasma at temperature $T=10 \mathrm{eV}$ and density $\rho=2.463 \mathrm{~g} / \mathrm{cc}$. The dotted and dashed curves represent contributions to the radial density for energies $p^{2} / 2 m+V(r)$ below and above zero, respectively. Right panel: The $p^{2} / 2 m+V(r)>0$ (continuum) contribution to $n_{e}(r)$ approaches a constant value $n_{e}$ at the cell boundary, interpreted as the average electron density of the plasma .

The solid curve in the upper panel of Fig. 2 shows the radial density $4 \pi r^{2} n_{e}(r)$ for a boron plasma at $T=10 \mathrm{eV}$ and density $2.463 \mathrm{~g} / \mathrm{cc}$. The contribution to the integral in Eq. (5) from energies $p^{2} / 2 m+V(r)<0$ are illustrated by the dotted curve. This contribution is the semi-classical counterpart of quantum mechanical bound states. The dashed curve shows the contribution to the radial density from energies $p^{2} / 2 m+V(r) \geq 0$. In the right panel, we divide out the factor $4 \pi r^{2}$ from the dashed curve and show that the continuum contribution to $n_{e}(r)$ smoothly approaches a constant value $n_{e}=n_{e}(R)$ at the WS cell boundary. The value $n_{e}$ is interpreted as the density of the uniform electron background in which the WS cell floats. To establish neutrality outside the WS cell, there must be a compensating ion background charge $Z^{*}=n_{e} / n_{i}$.

In the generalized Thomas-Fermi theory, the contribution of electrons to the pressure is

$$
p_{e}=\frac{(2 m k T)^{5 / 2}}{6 m \pi^{2}} I_{3 / 2}(\mu / k T)
$$

where

$$
I_{3 / 2}(x)=\int_{0}^{\infty} \frac{y^{3 / 2} d y}{\left[1+e^{y-x}\right]}
$$

The electron contribution to the potential energy per ion is

$$
\begin{aligned}
& e_{\mathrm{e}-\mathrm{pot}}= \int_{0}^{R} 4 \pi r^{2} \rho(r)\left(r \frac{d V}{d r}\right) d r \\
&=-\int_{0}^{R} 4 \pi r^{2} n_{e}(r) \frac{Z}{r} d r+\int_{0}^{R} 4 \pi r n_{e}(r) d r \int_{0}^{r} 4 \pi s^{2} n_{e}(s) d s \\
&=e_{e-n}+e_{e-e},
\end{aligned}
$$

and the electron contribution to the kinetic energy can be obtained from the 
Thomas-Fermi Hugoniot Curves
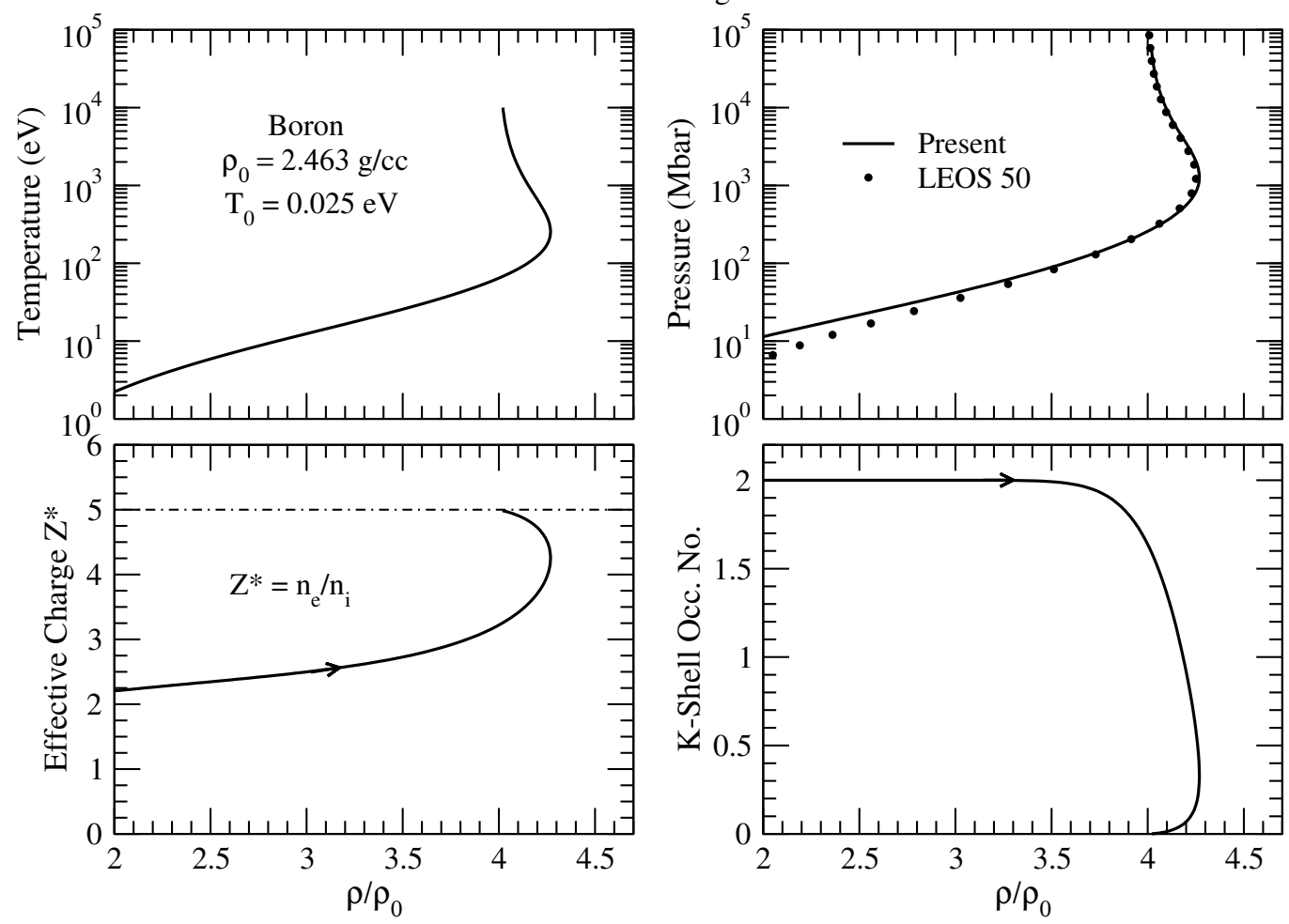

Figure 3: Upper-left panel: Solution to Hugoniot equation $T\left(\rho / \rho_{0}\right)$. Upper right: $p\left(\rho / \rho_{0}\right)$ obtained with the aid of the TF equation of state is shown by the solid line and LEOS 50 data from the Livermore LEOS database is shown by dots. Lower-left: Average ionic charge of the plasma $Z^{*}\left(\rho / \rho_{0}\right)=n_{e} / n_{i}$. Lower-right: K-shell occupation number determined by solving the Schrödinger equation in the TF potential.

"virial" theorem

$$
e_{\mathrm{e}-\mathrm{kin}}=\frac{3}{2} p V_{\mathrm{WS}}-\frac{1}{2} e_{\mathrm{e}-\mathrm{pot}}
$$

As mentioned earlier, the ideal gas laws:

$$
\begin{aligned}
p_{i} & =n_{i} k T \\
e_{i} & =\frac{3}{2} k T
\end{aligned}
$$

are used to determine the ion contributions to pressure and energy. This treatment leads to some error at low temperature, but is well justified in the temperature range $20-500 \mathrm{eV}$ of primary interest in the present study. The pressure and energy in the Hugoniot equation are the sum of the electron and ion contributions.

In the upper-left panel of Fig. 3. we show the solution $T(\mathrm{eV})$ vs. $\rho / \rho_{0}$ to the Hugoniot equation for a boron plasma initially at temperature $T_{0}=0.025 \mathrm{eV}$ 
and density $\rho_{0}=2.263 \mathrm{~g} / \mathrm{cc}$. With the aid of the plasma equation of state, the solution curve can be converted into the curve giving $p$ as a function of $\rho / \rho_{0}$ shown in the upper-right panel of the figure. The dotted LEOS 50 curve from the Livermore LEOS database shown in the upper right panel is obtained using an equation of state at low temperatures in which the the $\mathrm{TF}+$ ideal gas expressions for pressure and energy are modified to account for ionic bonding [16, 17. . Differences between these two pressure Hugoniot curves are found to have little influence on the opacity calculations in the present work. On the other hand, the pressure Hugoniot curves in Fig. 3 have a peak compressibility that is about $7 \%$ less compressed (stiffer than) recent path-integral Monte Carlo (PIMC) and density-functional-theory molecular-dynamics (DFT-MD) calculations [18 in the temperature interval where the opacity is changing rapidly! In the lower-left panel of Fig. 3. the average background ion charge $Z^{*}=n_{e} / n_{i}$ along the Hugoniot curve is shown. It is seen that as temperature increases $Z^{*}$ converges to 5 corresponding to complete ionization of boron ions in the plasma.

Finally, in the lower-right panel of Fig. 3, the K-Shell occupation number inferred by solving the Schrödinger equation in the TF potential is shown. The $\mathrm{K}$-shell occupation is given in terms of the $1 s$ eigenvalue $\epsilon_{1 s}$ by

$$
\text { occ no. }=\frac{2}{1+\exp \left[\left(\epsilon_{1 s}-\mu\right) / k T\right]} .
$$

The fact that the limiting high temperature values of all four functions illustrated in Fig. 4 occur at $\rho / \rho_{0}=4$ is a consequence of using the ideal gas law to describe the ionic motion.

\section{Average-Atom Model and K-shell occupation}

A quantum-mechanical average-atom cell model is used to test the accuracy of the Thomas-Fermi calculation of the K-shell occupation number described in the previous paragraph. The average-atom cell model differs from the ThomasFermi theory in two essential ways. Firstly, the expression for the potential $V(r)$ given in Eq. (4) is modified by including a (local) exchange-correlation correction:

$$
V(r)=-\frac{Z}{r}+\int_{r^{\prime} \leq R} \frac{n_{e}\left(\boldsymbol{r}^{\prime}\right) d^{3} r^{\prime}}{\left|\boldsymbol{r}-\boldsymbol{r}^{\prime}\right|}+V_{x c}\left(n_{e}\right) .
$$

Secondly, the semi-classical expression for $n_{e}(r)$ in Eq. (5) is replaced by the quantum-mechanical expression

$$
\begin{aligned}
n_{e}(r)=\frac{1}{4 \pi r^{2}}\left[\sum_{n l} \frac{2(2 l+1)}{1+\exp \left[\left(\epsilon_{n l}-\mu\right) / k T\right]} P_{n l}^{2}(r)\right. & \\
& \left.\quad+\sum_{i} \int_{0}^{\infty} d \epsilon \frac{2(2 l+1)}{1+\exp [(\epsilon-\mu) / k T]} P_{\epsilon l}^{2}(r)\right],
\end{aligned}
$$

where $P_{n l}(r)$ is the radial wave function for a bound-state with quantum numbers $n$ and $l$ and $P_{\epsilon l}(r)$ is the radial wave function for a continuum state with 

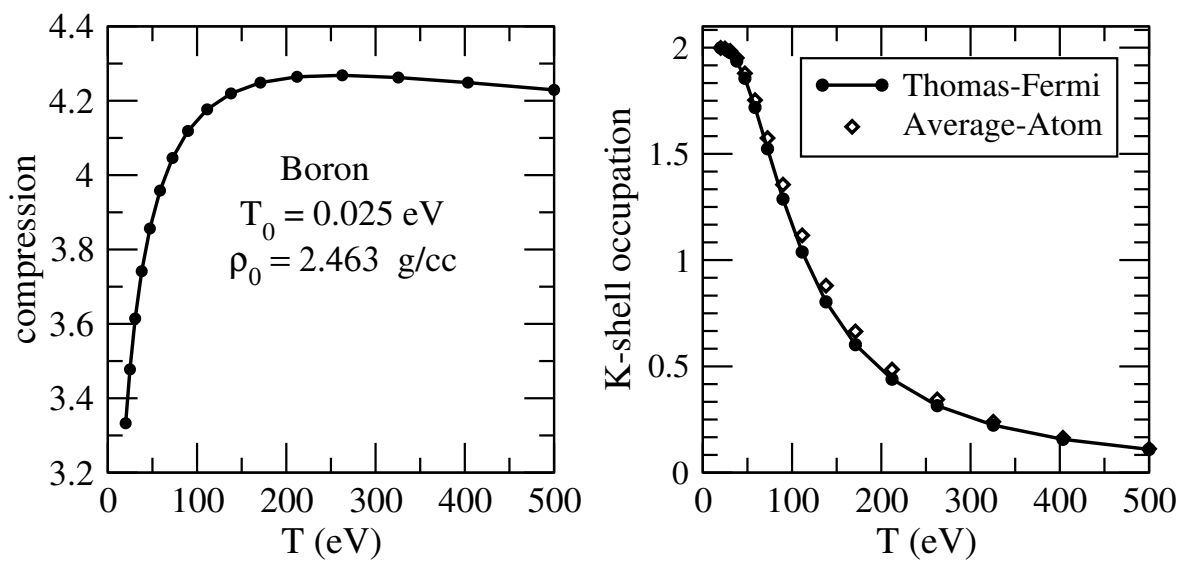

Figure 4: Left panel: Hugoniot relation giving the compression ratio in the hot plasma in terms of $T(\mathrm{eV})$. Dots designate comparison temperatures. Right panel: K-shell occupation from the average-atom model (diamonds) compared with values from the TF theory (dots).

energy $\epsilon$ and angular quantum number $l$. The continuum states are normalized on the energy scale. The TF requirement Eq. (6) that the WS cell be neutral remains unchanged in the average-atom theory.

The K-shell occupation predicted by the average-atom model (the number of bound electrons for the cases considered here where only the K-shell is occupied) is compared with values from the TF theory in Fig. 4 for temperatures ranging from $20 \mathrm{eV}$ to $500 \mathrm{eV}$ in the shock-heated plasma. In the left panel of Fig. 4, the Hugoniot curve relating the compression ratio to the temperature behind the shock is shown. The closed circles designate specific comparison temperatures. In the right panel, values of the K-shell occupation obtained from the TF theory are shown by the black curve and the dots. These TF values are compared with occupation numbers from the average-atom model which are marked by diamonds. The maximum relative difference between the two determinations is $10 \%$ and occurs near $170 \mathrm{eV}$. Other plasma properties, such as electron density and chemical potential, calculated in the average-atom approximation agree within $10 \%$ with the corresponding TF values. It is found, however, that calculating opacities using bound and continuum wave functions evaluated in the TF potential leads to unphysical oscillations at high photon energies; therefore, the average-atom approximation is used in the following sections to study opacities.

\section{Opacity: Atomic Scattering Factor}

An electromagnetic plane wave traveling in the $z$ direction that scatters from an ion acquires an outgoing spherical component

$$
e^{i k z-i \omega t} \rightarrow e^{i k z-i \omega t}+\frac{r_{0}}{r} e^{i k r-i \omega t} f(\theta) \cos \phi,
$$


where $\theta$ is the scattering angle and $f(\theta)$ defines the atomic scattering factor (a.k.a. scattering amplitude). In the above, $r_{0}$ is the classical electron radius, introduced to make the scattering factor dimensionless. The coefficient $\cos \phi$ ranges from 1, when the polarization directions of the incident and scattered wave are parallel, to 0 when they are perpendicular.

From Huygens's principle, it follows that the scattered components of a plane wave traveling in a medium add coherently to create a plane wave moving in the same direction but with index of refraction $n$ :

$$
n=1-\frac{r_{0} \lambda^{2}}{2 \pi} n_{i} f(0),
$$

where $\lambda$ is the wavelength of the electromagnetic wave, $n_{i}$ is the ion density and $f(0)$ is the forward scattering factor. The forward scattering factor $f(0)$, which is complex, is written

$$
f(0)=f_{1}-i f_{2} .
$$

As shown in Ref. 2, for x-ray energies greater than the photoionization threshold,

$$
\begin{aligned}
& f_{1} \approx Z, \\
& f_{2}=\frac{\sigma_{\mathrm{bf}}(\omega)}{2 r_{0} \lambda},
\end{aligned}
$$

where $\sigma_{\mathrm{bf}}(\omega)$ is the bound-free photoionization cross section. The relation between $f_{2}$, which is responsible for plasma opacity, and $\sigma_{\mathrm{bf}}(\omega)$ is a consequence of the optical theorem. It follows that the intensity of a plane electromagnetic wave traveling in the medium described above is

$$
I(z)=I_{0} \exp \left(-2 r_{0} \lambda n_{i} f_{2} z\right)=I_{0} \exp \left(-n_{i} \sigma_{\mathrm{bf}} z\right) .
$$

The quantum mechanical expression for photoionization of the K-shell of an atom or ion is

$$
\sigma_{\mathrm{bf}}(\omega)=\frac{8 \pi^{2}}{3} \alpha \omega|D|^{2},
$$

where $D$ is the dipole matrix element

$$
D=\int_{0}^{\infty} P_{\epsilon 1}(r) r P_{1 s}(r) d r .
$$

In the present study, we evaluate the cross section using average-atom wave functions. To account for the fact that the K-shell is partially occupied, the cross section must be multiplied by the fractional K-shell occupation: occ/2.

It is of interest to compare the photoionization cross section calculated using average atom wave functions with the corresponding neutral-atom cross section. To this end, the average-atom cross section for boron at $T=10 \mathrm{eV}$ and density $\rho=2.463 \mathrm{~g} / \mathrm{cc}$, where the $\mathrm{K}$-shell is fully occupied, is compared in the upper panel of Fig. 5 with the K-shell cross section for a neutral boron atom given by 


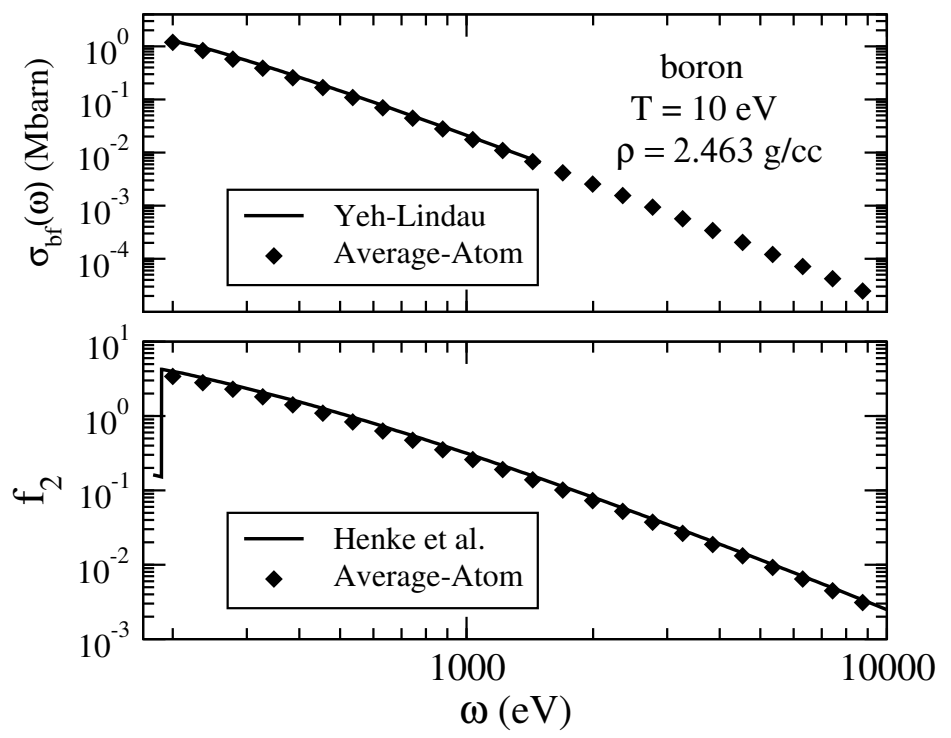

Figure 5: Upper panel: The average-atom K-shell photoionization cross section for a warmdense boron plasma at $T=10 \mathrm{eV}$ and $\rho=2.463 \mathrm{~g} / \mathrm{cc}$ in which the K-shell is fully occupied, shown by diamond symbols, is compared with the K-shell cross section for a neutral boron atom [1] shown in the black line. Lower panel: The average-atom atomic scattering factor $f_{2}$ for warm-dense boron, shown by the diamond symbols, is compared with $f_{2}$ for cold boron from the Henke table 2, shown in the black line. 


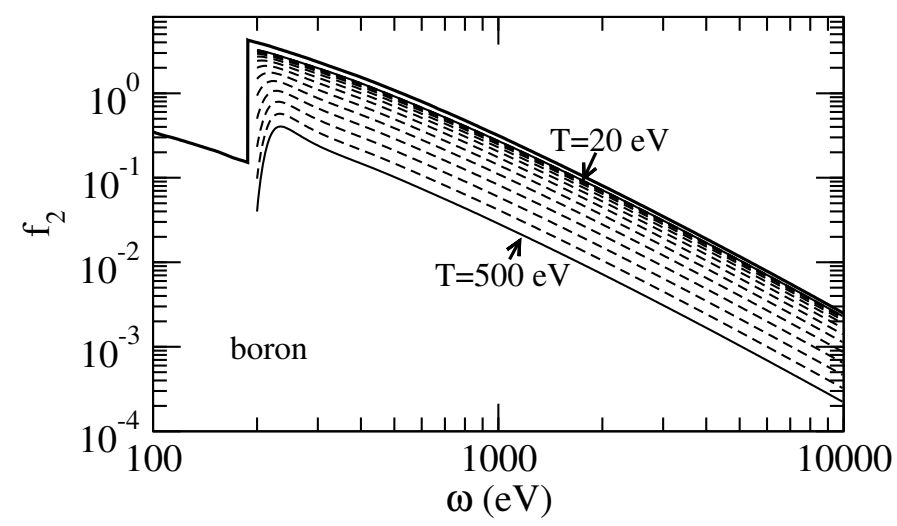

Figure 6: The (cold-matter) atomic scattering factor $f_{2}$ for boron from Ref. 2] (thick black curve) is compared with average-atom calculations of $f_{2}$ (dashed curves) carried out at temperatures between 20 and $500 \mathrm{eV}$ marked by the dots on the boron Hugoniot curve shown in the left panel of Fig. 4

Yeh and Lindau [1. In the lower panel of Fig. 5 , the value of $f_{2}$ obtained from Eq. (21) is compared with the cold-matter value tabulated in Ref. 2]. Both the average-atom cross section and scattering factor for this warm-dense plasma, where the K-shell is fully occupied, are found to be in close agreement with well-established cold matter results.

This situation changes as the temperature increases on the hot side of the plasma and the K-shell occupation decreases. In Fig. 6, we compare the Henke cold-matter result for $f_{2}$, shown by the black curve, with average-atom results for $f_{2}$, shown by the dashed curves. These curves are evaluated at the densities and temperatures marked out by the dots on the Hugonoit curve shown in the left panel of Fig. 4. As expected, the average-atom values of $f_{2}$ decrease systematically with temperature along the Hugoniot.

\section{Opacity: Mass Attenuation Coefficient}

An alternative measure of opacity is the x-ray mass attenuation coefficient $\mu / \rho$ defined and tabulated in Ref. 3. For single-ion plasmas, in the photon energy interval $10^{2} \mathrm{eV} \leq \omega \leq 10^{4} \mathrm{eV}$, where the x-ray opacity is governed by $\mathrm{K}$-shell photoionization, the attenuation coefficient is defined by $\mu=n_{i} \sigma_{\mathrm{bf}}$ for a single-ion plasma, leading to the result,

$$
I(z)=I_{0} \exp (-\mu z)
$$

for the reduction of intensity of an electromagnetic wave. It follows from the fact that the ion number density is related to the plasma density by

$$
n_{i}=\frac{N_{A}}{A} \rho
$$




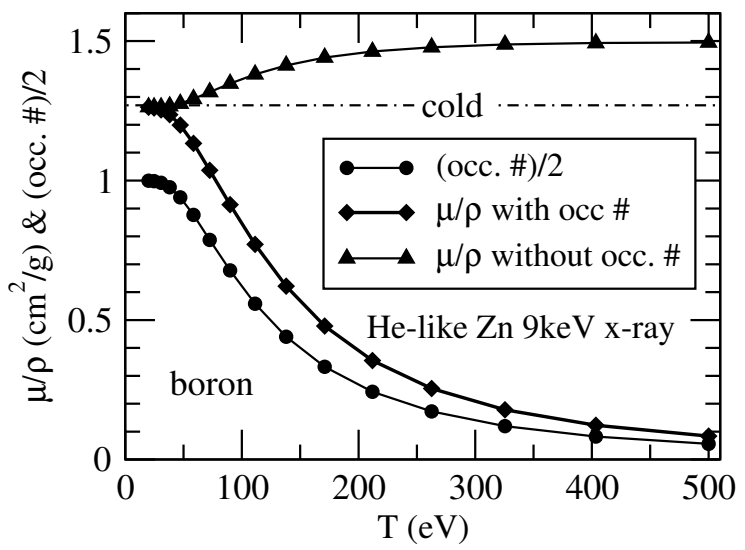

Figure 7: The boron mass attenuation coefficient $\mu / \rho$ for a $9 \mathrm{keV}$ He-like Zn x-ray and the relative K-shell occupation are plotted as functions of temperature along the boron Hugoniot. The circles give the relative K-shell occupation at points marked on the boron Hugoniot curve in Fig. 4 the diamonds show $\mu / \rho$ including the K-shell occupation, whereas, the triangles show the $\mu / \rho$ evaluated assuming a filled-K-shell. The occupation factor accounts for a $95 \%$ reduction in the opacity over the $20-500 \mathrm{eV}$ interval, while the photoionization cross section is responsible for an $18 \%$ increase.

that

$$
\mu / \rho=\frac{N_{a}}{A} \sigma_{\mathrm{bf}}
$$

depends on plasma properties only indirectly through the photoionization cross section.

For multi-ion plasmas, the expression for $\mu / \rho$ is

$$
\mu / \rho=\sum_{i} x_{i}(\mu / \rho)_{i},
$$

where $x_{i}$ is the concentration of ionic species $i$.

The relative importance of the K-shell occupation on the $\mu / \rho$ for a $9 \mathrm{keV}$ $\mathrm{x}$-ray is illustrated in Fig. 7. The curve marked by diamonds shows results of a calculation of $\mu / \rho$ in which the photoionization cross section given in Eq. (23) is multiplied by occ. $/ 2$, shown by dots, accounting for a $95 \%$ reduction of the opacity over the $20-500 \mathrm{eV}$ temperature interval. The curve in Fig. 7 marked by triangles shows that $\mu / \rho$ without the K-shell occupation factor actually increases by $18 \%$. This example illustrates the importance of understanding of the Kshell occupation, but adds the caution that the photoionization cross section also varies along the Hugoniot.

In Fig. 8, segments of the temperature Hugoniot curves between 20 and $500 \mathrm{eV}$ and the corresponding x-ray mass attenuation coefficient $\mu(T)$ for a $9 \mathrm{keV}$ $\mathrm{x}$-ray impinging on a shock-heated plasma at temperature $T$ and compression ratio $\rho / \rho_{0}$ illustrate the theory developed herein for materials $\mathrm{B}, \mathrm{C}, \mathrm{B}_{4} \mathrm{C}$ and $\mathrm{BN}$ mentioned in Ref. [18. 

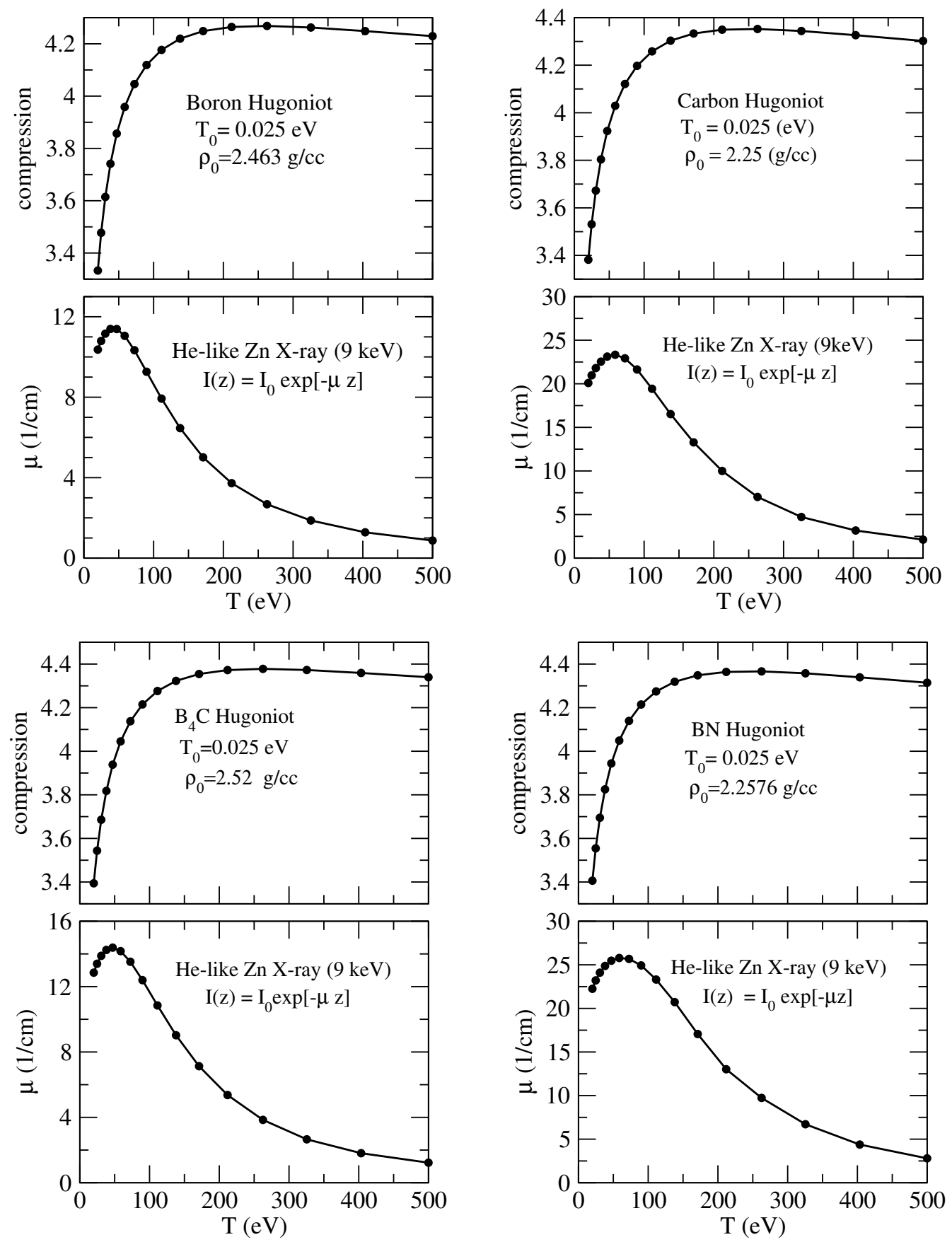

Figure 8: Segments of the temperature Hugonot are shown along with the x-ray mass attenuation coefficient $\mu$ for $\mathrm{B}, \mathrm{C}, \mathrm{B}_{4} \mathrm{C}$ and $\mathrm{BN}$ at points along the temperature Hugoniot. Material densities $\rho$ can be inferred from the compression ratios. The relative magnitude of $\mu(T)$ for these materials is a reflection of the strong $Z$ dependence of the K-shell photoionization cross section. 
Densities $\rho_{1}$ and $\rho_{2}$ of component ions in a two-component plasma with concentrations $x_{1}$ and $x_{2}$ and density $\rho$ are determined by requiring that the WS volume of an average ion in the plasma is the average of the WS volumes of the constituent ions:

$$
\frac{x_{1} A_{1}+x_{2} A_{2}}{N_{A} \rho}=x_{1} \frac{A_{1}}{N_{A} \rho_{1}}+x_{2} \frac{A_{2}}{N_{A} \rho_{2}},
$$

where $A_{1}$ and $A_{2}$ are the atomic weights of the ions and $N_{A}$ is Avogadro's constant. Additionally, we require that the free-electron densities associated with distinct ions be identical:

$$
n_{e}=n_{e}(1)=n_{e}(2) .
$$

Solving the two equations above simultaneously permits one to determine $\rho_{1}$ and $\rho_{2}$. It should be noted that the second condition also ensures that the pressure $p$ and chemical potential $\mu$ associated with each ion type, and consequently of the plasma, is unique. The electron energy in the plasma is just the weighted sum of the electron energies of the two components.

The function $\mu(T)$ shown in Fig. 8 increases with temperature near $T=$ $20 \mathrm{eV}$ because $\mu / \rho$ takes on its constant cold-matter value while $\rho$ increases with $T$ along the Hugioniot for low temperatures. The peak value of $\mu(T)$ for $\mathrm{B}_{4} \mathrm{C}$ is slightly larger than that for $\mathrm{B}$ owing to the $20 \%$ admixture of $\mathrm{C}$. Moreover, the peak values of $\mu(T)$ for $\mathrm{BN}$ is about twice as large as that for $\mathrm{B}$ and slightly larger than the peak value for $\mathrm{C}$ owing to the $50 \%$ admixture of N. The observed sensitivity of the peak values of $\mu(T)$ to ionic constituents can be traced to the strong $\left(Z^{5}\right)$ dependence of the photoionization cross section on ionic charge $Z$.

\section{Summary and Conclusions}

A systematic method for evaluating the opacity of shock-heated light-element plasmas has been developed and applied to boron, carbon, boron carbide and boron nitride. This method consists of evaluating the shock Hugoniot in the temperature interval where the K-shell occupation drops from two to near zero and evaluating the photoionization cross section for temperatures and densities

along the Hugoniot in the average-atom approximation. For (relatively) cold plasmas, where the K-shell is fully occupied, the average-atom theory leads to values of $f_{2}$ and $\mu / \rho$ very close to those tabulated in Refs. 2, 3. As temperature increases along the Hugoniot, the opacity decreases from the cold matter values. This decrease is found to be governed dominantly by the falloff of the K-shell occupation, but to a lesser extent by the dependence of the $\sigma_{\mathrm{bf}}$ on density and temperature along the Hugoniot.

\section{Acknowledgments}

The authors owe a debt of gratitude to Phil Sterne for an introduction to Hugoniot calculations in plasmas, to Brian Wilson for assistance on averageatom calculations and to Heather Whitley for advice on the equations of state 
for boron and boron compounds. The work of J.N. was performed under the auspices of the U.S. Department of Energy by Lawrence Livermore National Laboratory under Contract DE-AC52-07NA27344.

\section{References}

[1] J. J. Yeh, I. Lindau, Atomic Subshell Photoionization Cross Sections and Asymmetry Parameters $1<Z<103$, Atomic Data and Nuclear Data Tables 32 (1985) 1.

[2] B. I. Henke, E. M. Gullikson, J. C. Davis, X-ray Interactions: Photoabsorption, Scattering, Transmision, and Reflection a $\mathrm{E}=50-30,000 \mathrm{eV}, \mathrm{Z}=1-92$ ", Atomic Data and Nuclear Data Tables 54 (1993) 181.

[3] J. H. Hubbell, S. M. Seltzer, Xray Mass Attenuation Coefficients, http://physics.nist.gov/PhysRefData/Xray Mass Coef/cover.html.

[4] R. P. Feynman, N. Metropolis, E. Teller, Equations of state of elements based on the generalized Fermi-Thomas theory, Phys. Rev. 75 (1949) 15611573 .

[5] J.-M. Yuan, Opacity of hot and dense plasmas of a mixture using an average-atom approach, Chinese Phys. Letts. 19 (2002) 1459.

[6] B. F. Rozsnyai, Electron scattering in hot/warm plasmas, HEDP 4 (2008) 64-72.

[7] R. Piron, T. Blenski, Variational-average-atom-in-quantum-plasmas (VAAQP) code and virial theorem: Equation-of-state and shock-hugoniot calculations for warm dense $\mathrm{Al}, \mathrm{Fe}, \mathrm{Cu}$, and Pb, Phys. Rev. E 83 (2011) 026403.

[8] M. Murillo, J. Weisheit, S. Hansen, M. Dharma-wardana, Partial ionization in dense plasmas: Comparisons among average-atom density functional models, Phys. Rev. E 87 (2013) 063113.

[9] S. X. Hu, L. A. Collins, V. N. Goncharov, J. D. Kress, R. L. McCrory, S. Skupsky, First-principles equation of state of polystyrene and its effect on inertial confinement fusion implosions, Phys. Rev. E 92 (2015) 043104.

[10] M. Jeffery, J. W. O. Harris, D. J. Hoarty, On the Optimized Atomic Exchange Potential method and the CASSANDRA opacity code, HEDP 20 (2016) 1-8.

[11] N. Shaffer, N. Ferris, J. Colgan, D. Kilcrease, C. Starrett, Free-free opacity in dense plasmas with an average atom model, HEDP 23 (2017) 31-37.

[12] S. X. Hu, L. A. Collins, J. P. Colgan, V. N. Goncharov, D. P. Kilcrease, Optical properties of highly compressed polystyrene: An ab initio study, Phys. Rev. B 96 (2017) 144203. 
[13] D. Swift, A. Kritcher, J. Hawreliak, A. Lazicki, A. MacPhee, B. Bachmann, T. Döppner, J. Nilsen, G. Collins, S. Glenzer, S. Rothman, D. Kraus, R. Falcone, Absolute Hugoniot measurements from a spherically convergent shock using x-ray radiography, Rev. Sci. Instrum. 89 (2018) 053505.

[14] M. B. Trzhaskovskaya, V. K. Nikulin, Atomic structure data based on average-atom model for opacity calculations in astrophysical plasmas, HEDP 26 (2018) 1-7.

[15] R. Piron, T. Blenski, Average-atom model calculations of dense-plasma opacities: Review and potential applications to white-dwarf stars, Contributions to Plasma Physics 58 (1) (2018) 30-41.

[16] R. M. More, K. H. Warren, D. A. Young, G. B. Zimmerman, A new quotidian equation of state (QEOS) for hot dense matter, Phys. Fluids 31 (1988) 3059 .

[17] D. A. Young, E. M. Corey, A new global equation of state model for hot, dense matter, J. Appl. Phys. 78 (1998) 3748.

[18] S. Zhang, B. Militzer, M. Gregor, K. Caspersen, L. Yang, J. Gaffney, T. Ogitsu, D. Swift, A. Lazicki, D. Erskine, R. London, P. Celliers, J. Nilsen, P. Sterne, H. Whitley, Theoretical and experimental investigation of the equation of state of boron plasmas, Phys. Rev. E 98 (2018) 023205. 